\title{
Mitochondrial biogenesis and degradation are induced by CCCP treatment of porcine oocytes
}

\author{
N Itami, S Shiratsuki, K Shirasuna, T Kuwayama and H Iwata \\ Department of Animal Reproduction, Tokyo University of Agriculture, Funako 1737, Atsugi, Kanagawa, \\ 243-0034, Japan \\ Correspondence should be addressed to H Iwata; Email: h1iwata@nodai.ac.jp
}

\begin{abstract}
In this study, we investigated the mitochondrial quality control system in porcine oocytes during meiotic maturation. Cumulus cell oocyte complexes (COCs) collected from gilt ovaries were treated with $10 \mu \mathrm{M}$ carbonyl cyanide-m-chlorophenylhydrazone (CCCP; a mitochondrial uncoupler) for $\mathbf{2} \mathrm{h}$. The CCCP treatment was found to significantly reduce ATP content, increase the amount of phosphorylated AMP-activated protein kinase and elevate reactive oxygen species levels in oocytes. When the CCCP-treated COCs were cultured further for $44 \mathrm{~h}$ in maturation medium, the ATP levels were restored and the parthenogenetic developmental rate of oocytes to the blastocyst stage was comparable with that of untreated COCs. To examine the effects of CCCP treatment of oocytes on the kinetics of mitochondrial DNA copy number (Mt number), COCs treated with 0 or $10 \mu \mathrm{M} \mathrm{CCCP}$ were cultured for $44 \mathrm{~h}$, after which the Mt number was determined by RT-PCR. CCCP treatment was found to increase the Mt number in the modified maturation medium in which mitochondrial degradation was inhibited by MG132, whereas CCCP treatment did not affect the Mt number in the maturation medium lacking MG132. The relative gene expression of TFAM was furthermore shown to be significantly higher in CCCP-treated oocytes than in untreated oocytes. Taken together, the finding presented here suggest that when the mitochondria are injured, mitochondrial biogenesis and degradation are induced, and that these processes may contribute to the recuperation of oocytes.
\end{abstract}

Reproduction (2015) 150 97-104

\section{Introduction}

Mitochondrial quality is important for cellular health. In somatic cells, mitochondrial quality is maintained through the removal of damaged mitochondrial components by mitochondrial proteases as well as through mitochondrial dynamics, including fusion, fission and mitophagy (Tatsuta \& Langer 2008, Chen et al. 2012). On the other hand, mitochondrial quality-control systems have not been extensively studied in oocytes. Mitochondrial quality and quantity are believed to be important for oocyte maturation, fertilization and subsequent development (Reynier et al. 2001, Thouas et al. 2004, Santos et al. 2006, Shourbagy et al. 2006). It has been reported that the mitochondrial number increases during oocyte development (Cotterill et al. 2013). Early embryonic development may be supported by whole, already existing mitochondria without de novo synthesis (Wai et al. 2010), although a few studies suggest that de novo mitochondrial synthesis occurs during early embryonic development (Chiaratti et al. 2010). Furthermore, it has been suggested that once mitochondrial number reaches a certain threshold during oocyte maturation, this number is maintained (Mahrous et al. 2012, Cotterill et al. 2013), but Mao et al. (2012) reported that mitochondrial synthesis is induced by supplementation of maturation medium with follicular fluid (FF) and neuregulin 1. Despite the presence of both de novo synthesis and degradation pathways in somatic cells, mitochondrial turnover during oocyte maturation has not been investigated. In this context, Sato et al. (2014) reported that when oocytes were cultured in a medium containing a proteasome inhibitor, mitochondrial DNA copy number (Mt number) increased during oocyte maturation, suggesting that proteasomes play a role in mitochondrial removal. Using the abovementioned culture conditions, the authors further showed that resveratrol upregulates mitochondrial biosynthesis. Resveratrol is an activator of SIRT1, which is a member of the sirtuin family, and NAD + dependently deacethylates versatile transcription factors related to cellular metabolism. SIRT1 and AMP-activated protein kinase (AMPK) activate PGC1 $\alpha$ by deacethylation and phosphorylation, resulting in mitochondrial biosynthesis through NRF1 and mitochondrial transcription factor A (TFAM) (Price et al. 2012). In addition, SIRT1 and AMPK play a role in autophagy (Wu et al. 2011) Treatment of cells with carbonyl cyanide- $m$-chlorophenylhydrazone (CCCP), an inhibitor of oxidative phosphorylation, induces autophagy to remove damaged mitochondria 
(Ding et al. 2010, Lee et al. 2010, Cali et al. 2013). In our study, we investigated whether a mitochondrial qualitycontrol system is present in oocytes by investigating mitochondrial function, DNA copy number and expression of genes related to mitochondrial generation and oocyte parthenogenetic developmental abilities, following CCCP-induced mitochondrial dysfunction, on the premise that induced dysfunction would upregulate mitochondrial turnover during oocyte maturation.

Porcine oocytes were treated with CCCP, after which ATP content, AMPK activity and subsequent parthenogenetic developmental ability were examined. Real-time PCR was furthermore used to assess the effects of CCCP treatment on the kinetics of the Mt number and the expression levels of genes associated with mitochondrial synthesis during oocyte maturation.

\section{Materials and methods}

\section{Chemicals and media}

All chemicals used in this study were purchased from Nacalai Tesque (Kyoto, Japan) unless otherwise indicated. The medium used for in vitro maturation (IVM) was North Carolina State University's 23 (NCSU23) solution (Petters \& Wells 1993) containing follicle fluid $(10 \% \mathrm{v} / \mathrm{v})$. The medium used for in vitro culture (IVC) was PZM-3 (Yoshioka et al. 2002). FFs were collected from antral follicles (3-6 $\mathrm{mm}$ in diameter), centrifuged (10 $000 \mathrm{~g}$ for $5 \mathrm{~min}$ ) and stored at $-30^{\circ} \mathrm{C}$. CCCP (SigmaAldrich) and MG132 (Sigma-Aldrich) were dissolved in DMSO at a concentration of $10 \mathrm{mM}$ ( $\times 1000$ of final concentration).

\section{Collection of ovaries and cumulus cell oocyte complexes}

The ovaries were collected from prepubertal gilts at a local slaughterhouse and transported to the laboratory within $1 \mathrm{~h}$ at $37^{\circ} \mathrm{C}$ in PBS containing antibiotics. Cell oocyte complexes (COCs) were retrieved from antral follicles, measuring 3-6 mm in diameter, using a $21 \mathrm{G}$ needle connected to a $5 \mathrm{ml}$ syringe. Oocytes with multiple compact granulosa layers and even cytoplasm were selected and pooled. Randomly selected COCs were used for each experiment, except for experiment 3, in which the mitochondrial DNA copy number was assessed.

\section{CCCP treatment, IVM, activation and IVC}

COCs were cultured in an IVM medium containing $0 \mu \mathrm{M}$ (vehicle, DMSO) or $10 \mu \mathrm{M} \mathrm{CCCP}$ for $2 \mathrm{~h}$ and were then washed in an IVM medium before being subjected to experiments.

For IVM, COCs were cultured in an IVM medium containing $1 \mathrm{mM}$ dibutyryl cAMP (dbcAMP), $10 \mathrm{IU} / \mathrm{ml}$ equine chorionic gonadotropin (ASKA Pharma Co. Ltd, Tokyo, Japan) and $10 \mathrm{IU} / \mathrm{ml}$ human chorionic gonadotropin (Fuji Pharma Co. Ltd, Tokyo, Japan) for $20 \mathrm{~h}$, after which the oocytes were transferred to an IVM medium that lacked dbcAMP and the hormones, and were cultured for $24 \mathrm{~h}$. After the IVM culture period, the oocytes were denuded from the surrounding cells and activated in an IVC medium containing $10 \mu \mathrm{g} / \mathrm{ml}$ ionomycin (Calbiochem, La Jolla, CA, USA) for $5 \mathrm{~min}$, and then cultured in an IVC medium containing $10 \mu \mathrm{g} / \mathrm{ml}$ cytochalasin B and $10 \mu \mathrm{g} / \mathrm{ml}$ cycloheximide for $6 \mathrm{~h}$. The oocytes were cultured in an IVC medium for 7 days, after which the rate of blastulation and the total blastocyst cell number were assessed. To determine the cell number, embryos were fixed in $4 \%$ paraformaldehyde, mounted on glass slides using an antifade reagent containing DAPI (ProLong gold antifade reagent with DAPI; Invitrogen) and observed under a digital fluorescence microscope (BZ-8000; Keyence, Tokyo, Japan). IVM was performed at $38.5^{\circ} \mathrm{C}$ in an atmosphere of $5 \%$ $\mathrm{CO}_{2}$ and $95 \%$ air, while IVC was performed at $38.5^{\circ} \mathrm{C}$ in an atmosphere of $5 \% \mathrm{O}_{2}, 5 \% \mathrm{CO}_{2}$ and $90 \% \mathrm{~N}_{2}$.

\section{Mitochondrial DNA copy number determination}

The Mt number in mature oocytes was determined by examining a group of ten oocytes collected from each donor. The oocyte number used for Mt number assays was based on previous reports that demonstrated that the Mt number determined from ten oocytes collected from a donor closely resembles the Mt number obtained by another group of ten cohort oocytes collected from the same donor (Sato et al. 2014). Oocytes were denuded from granulosa cells and the DNA extraction and PCR protocols procedures were performed according to previously described methods (Iwata et al. 2011). The Mt number was determined by performing real-time PCR using a Rotor-Gene 6500 real-time rotary analyser (Corbett Research, Mortlake, NSW, Australia) with the primers $5^{\prime}$-CGAGAAAGCACTTTCCAAGG-3' (forward) and $5^{\prime}$-CTAATTCGGGTGTTGGTGCT-3' (reverse) and Bio-Rad Ssofast-TM EvaGreen Supermix (Bio Rad). The primers were designed using Primer3Plus (http://sourceforge.net/projects/ primer3/) and sequence data for porcine mitochondria (accession number AF304202) to amplify a 151-bp region, from 8744-8314. The melting curve was analysed to verify the specificity of the PCR products, followed by electrophoresis to determine product size. As an external standard, the PCR product of the corresponding gene was cloned into a vector using the Zero Blunt TOPO PCR cloning kit (Invitrogen), the product of which was sequenced for confirmation before use. The amplification efficiencies of all PCR runs were $>1.9$.

\section{Quantitative $R$ T real-time PCR}

RNA was extracted from oocytes using an RNA isolation kit (RNAqueous-Micro, Ambion, Applied Biosystems) according to the manufacturer's instructions. At $20 \mathrm{~h}$ after CCCP treatment, 50 oocytes were randomly selected. The time point used was based on preliminary experiments. The extracted RNA was reverse-transcribed to cDNA using the Thermo script RT-PCR system (Invitrogen) according to the manufacturer's instructions. Primers used for RT were oligo $(\mathrm{dT})_{20}$ included in the kit. Quantification of cDNA was then performed by real-time PCR using the Rotor-Gene 6500 system. The primer sets used for the quantification of B-Actin, GAPDH, PGC1 $\alpha$ and TFAM were designed using DDBJ (http:// arsa.ddbj.nig.ac.jp/top-j.html) and Primer3 (http://frodo.wi. mit.edu/primer3/) and are listed in Table 1. Each PCR was 
Table 1 RT-PCR used this primer sequences.

\begin{tabular}{lllc}
\hline Gene & Primer & Accession no. & Amplicon size (bp) \\
\hline PGC1a & F: TTCCGTATCACCACCCAAAT & NW_213963 & 137 \\
& R: ATCTACTGCCTGGGGACCTT & & 164 \\
FFAM & F: GGCAGACTGGCAGGTGTA & NW_001130211 & \\
& R: CGAGGTCTTTTTGGTTTCCA & & 236 \\
B-Actin & F: GAGTCCACTGGTGTCTTCACG & NM_001206359 & \\
& R: ATGAGTCCCTCCACGATGC & & 179 \\
& F: ATCGTGCGGGACATCAAGGA & XM_003124280 & \\
\hline
\end{tabular}

PGC1a, Peroxisome proliferator-activated receptor gamma coactivator 1-alpha (PPARGC1a); TFAM,

Mitochondrial transcription factor A; GAPDH, Glyceraldehyde 3-phosphate dehydrogenase; B-Actin, $\beta$-actin.

performed with an initial denaturation step of $95^{\circ} \mathrm{C}$ for $1 \mathrm{~min}$ followed by 40 cycles of $95{ }^{\circ} \mathrm{C}$ for $5 \mathrm{~s}$ and $56{ }^{\circ} \mathrm{C}$ for $11 \mathrm{~s}$. SYBR green fluorescence was measured at the end of each extension step $\left(56^{\circ} \mathrm{C}\right)$. A melting curve was analysed to check the specificity of the PCR products, and agarose gel electrophoresis was carried out to check the resulting product sizes. Relative gene expression levels of each gene of interest were calculated by normalising to the expression levels of the endogenous controls $B$-Actin and GAPDH. Reactions were run in duplicate and experiments were repeated six times with different oocyte series. For each PCR run, a standard curve was generated using a tenfold dilution series of the corresponding standard plasmid of a known concentration.

\section{ATP measurement}

Oocytes were denuded from the granulosa cells and the ATP content of individual oocytes was determined by measuring the luminescence generated in an ATP-dependent luciferin-luciferase bioluminescence assay (ATP assay kit; Toyo-Inc., Tokyo, Japan) as described previously (Iwata et al. 2011). Each sample was prepared by adding individual oocytes to $50 \mu \mathrm{l}$ distilled water.

\section{Immunostaining detection}

Oocytes were denuded from the granulosa cells 8 or $20 \mathrm{~h}$ after CCCP treatment and immunostained as described previously (Takeo et al. 2013). Rabbit polyclonal anti-phospho-AMPK $\alpha$ (Thr172) (1:1000; Cell Signaling Technology, Inc., Beverly, MA, USA) was used as a primary antibody and goat anti-rabbit IgG FITC-conjugate was used as a secondary antibody (1:1000; Millipore, Tokyo, Japan). The oocytes were mounted onto glass slides with antifade reagent containing DAPI. The fluorescence intensity of p-AMPK in oocytes was observed using a digital fluorescence microscope (BZ-8000; Keyence) and quantified using ImageJ Software (NIH, Bethesda, MD, USA).

\section{Reactive oxygen species content determination in oocytes}

Oocytes were denuded from surrounding cells and incubated in an IVM medium containing CM-H2DCFDA (a general oxidative stress indicator; Life Technologies) for 30 min according to the manufacturer's instruction. Oocytes were then mounted on glass-bottomed slides and observed under a digital fluorescence microscope (Keyence). Fluorescence intensity of the oocytes was quantified using ImageJ Software (NIH).

\section{Experimental design}

Experiment 1

Effects of CCCP treatment on mitochondrial functions in oocytes were investigated. Oocytes were treated with 0 (vehicle control) or $10 \mu \mathrm{M}$ CCCP for $2 \mathrm{~h}$, immediately after which ATP and reactive oxygen species (ROS) content in the oocytes were measured. Experiments were each repeated at least twice (four times for ATP and twice times for ROS measurement) and the ATP and ROS contents were compared between the control and treated (10 $\mu \mathrm{M}$ CCCP) oocytes.

\section{Experiment 2}

Effects of CCCP treatment on subsequent oocyte viability were investigated. After $2 \mathrm{~h}$ of 0 (vehicle control) or $10 \mu \mathrm{M}$ CCCP treatment, 20 oocytes were cultured for $44 \mathrm{~h}$ in an IVM medium. Then ATP and ROS contents in the oocytes were measured, or oocytes were activated to allow for the developmental rate of oocytes to the blastocyst stage to be assessed 7 days after activation. The measurements of ATP and ROS content and the parthenogenetic developmental rate were repeated four, three and nine times respectively with different ovary series.

\section{Experiment 3}

Effects of CCCP treatment on the Mt number of oocytes were examined. Mitochondrial DNA copy numbers differ a great deal among donors, but the Mt number predicted by ten oocytes closely reflects the Mt number of other cohort oocytes collected from the same donors in cows and pigs (Iwata et al. 2011, Sato et al. 2014). Thus, in the present experiments, 20 oocytes were collected from a donor and divided into two groups: ten oocytes were treated with $10 \mu \mathrm{M}$ CCCP and the other ten were treated with $0 \mu \mathrm{M}$ CCCP (vehicle control). The oocytes were then washed and subjected to IVM, after which the Mt numbers in the oocytes were compared between the two groups. To highlight mitochondrial biogenesis during IVM, two cultures were used: standard IVM medium and IVM medium containing the proteasome inhibitor MG132. The addition of MG132 to the IVM medium inhibits protein degradation, as previously confirmed by the accumulation of ubiquitinated proteins in oocytes analysed by western blotting (Sato et al. 2014) as well as 
by immunostaining against ubiquitinated proteins in the present study (Supplementary Figure 1, see section on supplementary data given at the end of this article). In the medium containing MG132, mitochondrial degradation is also inhibited and culturing oocytes in the medium for $44 \mathrm{~h}$ thus significantly increased the Mt number (Sato et al. 2014). In the first experiment, control and CCCP-treated oocytes were cultured in an IVM medium and 16 donor gilts were used. In the subsequent experiment, control and CCCP-treated oocytes were cultured in an IVM medium containing MG132, and 15 donor gilts were used. Mt numbers were compared between the untreated (vehicle) and the $10 \mu \mathrm{M}$ CCCP-treated oocytes.

\section{Experiment 4}

This experiment was carried out to examine effects of CCCP treatment on mitochondrial synthesis by examining the expression levels of genes related to mitochondrial biogenesis. COCs were treated with 0 (vehicle control) or $10 \mu \mathrm{M}$ CCCP for $2 \mathrm{~h}$, before being cultured for a further $20 \mathrm{~h}$. Twenty hours was selected according to our preliminary experiments. Oocytes were denuded and used for RT-PCR analysis, in which the relative gene expression levels of PGC1a and TFAM (to those of $B$-Actin and GAPDH) were examined. This experiment was repeated six times, each with 50 oocytes collected from different ovary series.

\section{Experiment 5}

In this experiment, we examined the effect of CCCP treatment on the amount of phospho-AMPK. COCs were treated with 0 (vehicle control) or $10 \mu \mathrm{M}$ CCCP for $2 \mathrm{~h}$, after which oocytes were incubated in an IVM medium for 8 or $20 \mathrm{~h}$. The oocytes were then denuded and subjected to immunostaining. This experiment was repeated three times.

\section{Statistical analysis}

All data obtained in the present study were assessed using a Student's $t$-test, and developmental rates were arcsinetransformed prior to the statistical analysis. Differences with $P$ values $<0.05$ were considered statistically significant.
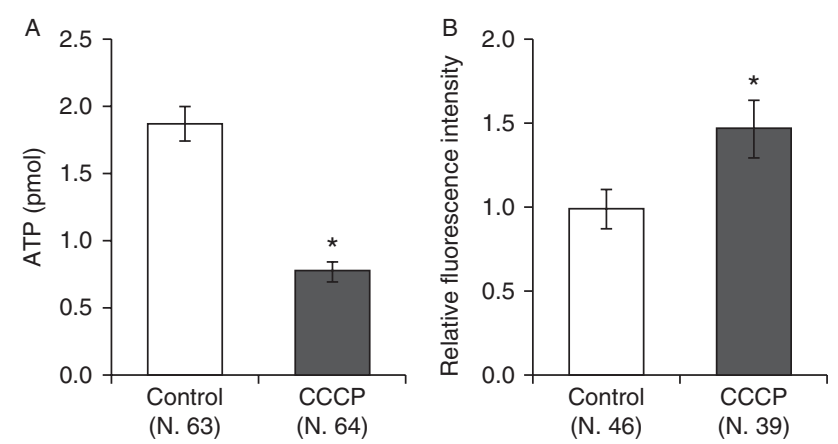

Figure 1 Effects of CCCP treatment of porcine oocytes on mitochondrial function. ATP (A) and ROS (B) content in oocytes after $2 \mathrm{~h}$ of CCCP treatment. The average fluorescence intensity of the control group in (B) was defined as 1.0. Data represent means \pm s.E.M. of three replicates. ${ }^{*} P<0.05$.
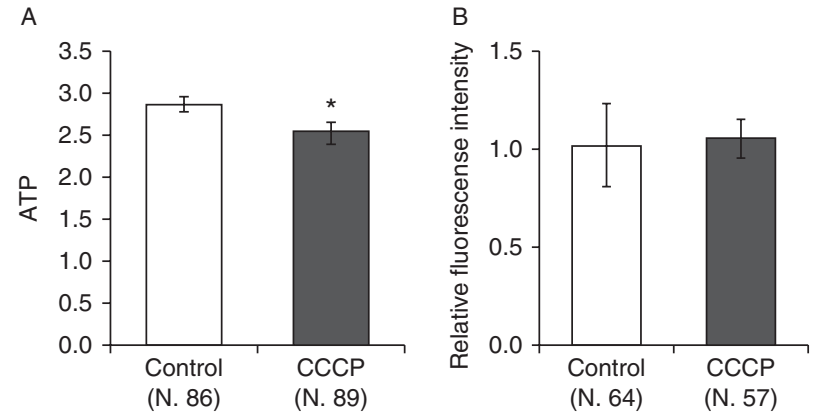

Figure 2 Effects of CCCP treatment of porcine oocytes on ATP contents after in vitro maturation (IVM). Oocytes were treated with CCCP for $2 \mathrm{~h}$ and ATP content was measured after $44 \mathrm{~h}$ of IVM. Data are represent means \pm S.E.M. ${ }^{*} P<0.05$.

\section{Results \\ CCCP treatment increases ROS generation and decreases ATP content in oocytes}

The effects of CCCP treatment on mitochondrial function were examined. As seen in Fig. 1 and 2-h CCCP treatment significantly reduced ATP content (A) to onethird of the level measured in untreated oocytes (1.81 pmol/oocyte vs 0.69 pmol/oocyte) and increased the ROS content in oocytes (B) by 1.49 \pm 0.17 -fold $(P<0.05)$ compared with untreated oocyte ROS levels.

\section{Effect of CCCP treatment on mitochondrial function was still observed after maturation but did not affect parthenogenetic development of oocytes}

CCCP-treated oocytes were further cultured for $44 \mathrm{~h}$ after which the ATP and ROS contents in mature oocytes and the parthenogenetic developmental rate of oocytes to blastocysts were examined. The difference between the ATP contents of the $10 \mu \mathrm{M}$ CCCP-treated and untreated oocytes decreased after IVM; however, the difference remained significant (Fig. $2 \mathrm{~A}, 2.53 \pm 0.12 \mathrm{pmol}$, vs $2.88 \pm 0.09 \mathrm{pmol})$. On the other hand, no difference in the levels of ROS was observed between CCCP-treated and vehicle-treated oocytes (Fig. 2B, 1.04-fold). The rates of oocyte parthenogenetic development to blastocysts were comparable between the two groups (Table 2, $13.7 \%$ vs $13.0 \%$ and 46.4 cells vs 50.8 cells; $P=0.86$ and $P=0.50$, respectively).

\section{CCCP treatment induces increases in mitochondrial DNA copy number in oocytes}

Mt number in untreated oocytes varied to a great extent among donors, with numbers ranging from 100080 to 529200 (Fig. 3A). The average Mt number in oocytes of all donors was 263191 for untreated oocytes and treatment with CCCP was found to decrease the Mt numbers to 197509 , a slight and insignificant $(P=0.12)$ decrease. Mt numbers are maintained by de novo 
Table 2 Effect of CCCP on oocytes developmental competence.

\begin{tabular}{lccccc}
\hline $\begin{array}{l}\text { CCCP concentration } \\
(\mu \mathrm{M})\end{array}$ & $\begin{array}{c}\text { No. of } \\
\text { replicates }\end{array}$ & $\begin{array}{c}\text { No. of } \\
\text { oocytes }\end{array}$ & $\begin{array}{c}\text { No. of } \\
\text { blastocysts }\end{array}$ & $\begin{array}{c}\text { Rate of blastulation } \\
\text { (mean } \pm \text { s.E.M.\%) }\end{array}$ & $\begin{array}{c}\text { Total cell number } \\
(\text { mean } \pm \text { s.E.M. \%) }\end{array}$ \\
\hline 0 & 9 & 176 & 24 & $13.7 \pm 3.2$ & $46.4 \pm 4.6$ \\
20 & 9 & 176 & 23 & $13.0 \pm 1.4$ & $50.8 \pm 4.4$ \\
\hline
\end{tabular}

synthesis and degradation, and thus CCCP-treated oocytes were cultured in the presence of MG132. In this experiment, the average Mt number in CCCP-treated oocytes was significantly greater than that in untreated oocytes: 249071 vs 183733 ( $P<0.02$, Fig. 3B).

\section{CCCP treatment induces increases in the expression of TFAM}

Effects of CCCP treatment on the expression levels of $P G C 1 \alpha$ and TFAM were assessed by RT-PCR and the resulting expression levels were normalised to those of $B$-Actin and GAPDH. As shown in Fig. 4, the relative expression level of PGC1a in oocytes was found to be slightly increased in CCCP-treated oocytes; however, the observed difference was not found to be statistically significant. TFAM expression, on the other hand, was significantly higher $(\sim 1.5$-fold $)$ in CCCP-treated oocytes than in untreated oocytes (A, B-Actin-normalised data; B, GAPDH-normalised data).

\section{CCCP treatment induces increases in the amount of phospho-AMPK in oocytes}

CCCP treatment was found to significantly increase the amount of phospho-AMPK at both the time points assessed (8 and $20 \mathrm{~h}$ of IVM; Fig. 5A, B and C).

\section{Discussion}

The findings of the present study show that when mitochondria in porcine oocytes are damaged, mitochondrial biogenesis and degradation are induced during oocyte maturation.

CCCP is a chemical inhibitor of oxidative phosphorylation, and according to a previous study, treatment of porcine oocytes with CCCP for $2 \mathrm{~h}$ results in a reduction in the ATP content in the oocytes by two-thirds, concomitant with a twofold increase in the ROS content of the oocytes (Cui et al. 2011). In agreement with the report by Cui et al. (2011), it was shown in the present study that treatment of oocytes with CCCP for $2 \mathrm{~h}$ significantly reduced ATP levels to $38 \%$ of the level observed in untreated oocytes and increased ROS levels by 1.5 -fold, indicating that CCCP treatment of oocytes induced mitochondrial dysfunction. After a further $44 \mathrm{~h}$ of culture, the ATP content in CCCP-treated oocytes was found to be restored to $88 \%$ of the levels measured in untreated oocytes, and no difference was observed in the level of ROS in oocytes between the CCCP- and vehicle-treated groups. No differences were observed in the parthenogenetic developmental rates of oocytes to the blastocyst stage and cell numbers in the blastocysts between the CCCP-treated and untreated oocytes groups. The results indicate that some aspects of mitochondrial damage are restored during oocyte maturation or that either partial or moderate disruption of mitochondrial function does not severely affect oocyte parthenogenetic development. Lee et al. (2014) reported that when porcine oocytes were treated with FCCP, a mitochondrial uncoupler, throughout the maturation period, the FCCP treatment resulted in a reduction of the mitochondrial number and in the developmental ability of oocytes. These contrasting results are likely due to overly long periods of FCCP treatment.
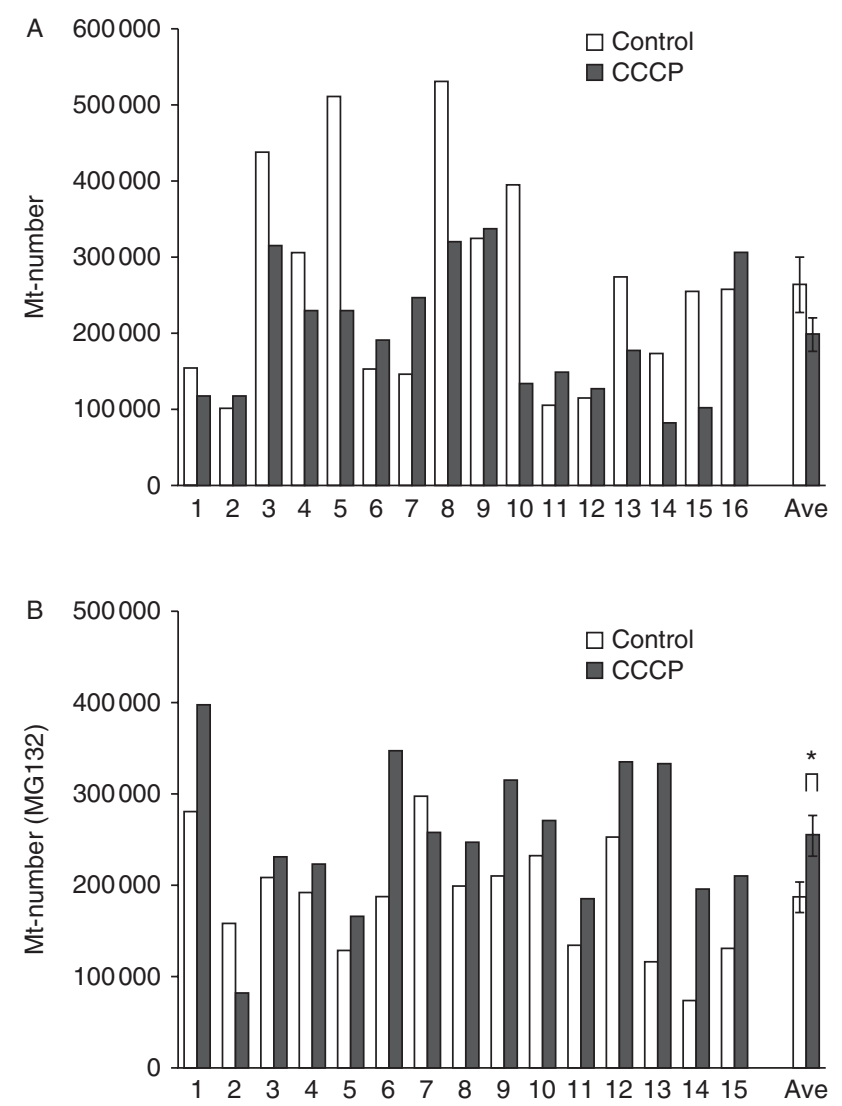

Figure 3 Effects of CCCP treatment on mitochondrial DNA copy number (Mt number) in oocytes. Oocytes were treated with 0 (control) or $10 \mu \mathrm{M} \mathrm{CCCP}$ for $2 \mathrm{~h}$ and the Mt numbers of oocytes were measured after IVM (44 h). Oocytes were either incubated in standard IVM medium (A) or in IVM medium containing MG132 (B). Data represent means \pm S.E.M. ${ }^{*} P<0.01$. 

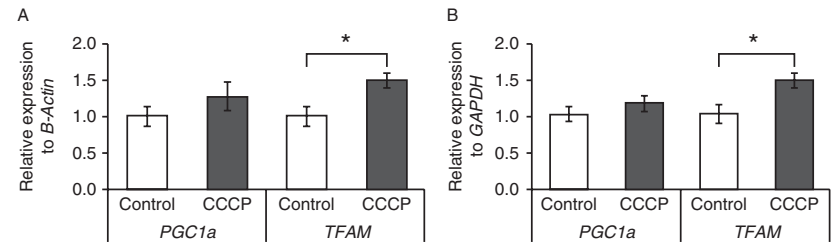

Figure 4 Effects of CCCP treatment of porcine oocytes on the expression of genes involved in mitochondrial biogenesis. The relative expression levels of PGC1a and TFAMnormalized to those of B-Actin (A) and $G A P D H(B)$ were measured. Values measured for untreated oocytes were defined as 1.0. Data represent means \pm S.E.M. ${ }^{*} P<0.05$.

The kinetics of the Mt number in oocytes are not yet fully understood. Mahrous et al. (2012) suggested that the Mt number in mouse oocytes remains constant once the oocytes reach full size. By contrast, the Mt number in porcine oocytes was found to increase during maturation period (Mao et al. 2012, Pawlak et al. 2012). From the above-mentioned reports it seems that the Mt number in oocytes may change under certain conditions and that the presence of both mitochondrial biogenesis and degradation pathways may mask the intrinsic mitochondrial kinetics. Spikings et al. (2007) and Pawlak et al. (2014) showed that when porcine oocytes were collected from antral follicles measuring 3-6 mm in diameter and divided into full-grown and immature oocytes, according to the results of brilliant cresyl blue staining, mitochondrial number only increased in oocytes categorised as developing oocytes. This result indicates that mitochondrial kinetics depend on the oocyte developmental stage, which may reflect the progression of the follicle phase in individual donors. In our previous reports, mitochondrial number determined using ten oocytes was almost identical to that determined using other cohorts of ten oocytes derived from the same donor gilts, at both the GV and M2 stages (Sato et al. 2014), indicating that comparison within cohort oocytes may avoid the bias by mixing oocytes from differential follicular phase. Sato et al. (2014) collected 20 oocytes from individual donor gilts, divided them into two groups and cultured the oocytes in a medium containing a proteasome inhibitor (MG132) or vehicle. They found that culturing oocytes with MG132 results in both the accumulation of ubiquitinated proteins and an increase in the Mt numbers of oocytes, through inhibition of mitochondrial degradation. In line with this, we demonstrated that culturing oocytes in MG132-containing medium and treatment of oocytes with CCCP both significantly increased the amount of ubiquitinated protein in oocytes (Supplementary Figure 1), indicating that MG132 does inhibit proteasome function and that CCCP treatment does induce protein degradation. Interestingly, the treatment of oocytes with CCCP in this study was found to increase Mt numbers in the MG132-containing IVM medium, whereas the Mt number in oocytes was slightly decreased by CCCP treatment in the medium lacking MG132. These observations suggest that mitochondrial damage caused by CCCP treatment is eliminated by the proteasome and that these mitochondria are replaced by de novo-synthesised mitochondria during oocyte maturation. Ge et al. (2012) reported that FCCP treatment of oocytes decreases maturation ability and spindle stability but does not alter the Mt number. We speculate that the reported result may be due to the presence of both pathways of mitochondrial biogenesis and degradation. The notion that CCCP treatment results in the upregulation of mitochondrial biogenesis is supported by the reported significant increases in the expression of TFAM, a key regulator of mitochondrial biogenesis (Smith et al. 2005, Wai et al. 2010, Wenz 2013). The Mt number is increased subsequently to TFAM expression (Spikings et al. 2007). PGC1 $\alpha$ is a key regulator of Mt biosynthesis, however, in the present study, gene expression level of $P G C 1 \alpha$ is not affected by CCCP treatment. This may be due to the fact that activation of PGC1 $\alpha$ needs posttranslational modifications including phosphorylation by AMPK and deacetylation by SIRT1 (Brenmoehl \& Hoeflich 2013). One question, however, remains: how are damaged mitochondria removed from oocytes? CCCP treatment has been used to induce mitophagy in cells (Ding et al. 2010, Chan et al. 2011, Ding et al. 2012), and autophagic clearance of selective damaged mitochondria will thus be investigated in future experiments.

AMPK is an energy sensor that interacts with SIRT1 to activate PGC1a and TFAM (Kwon et al. 2011). We previously reported that the treatment of porcine oocytes
A
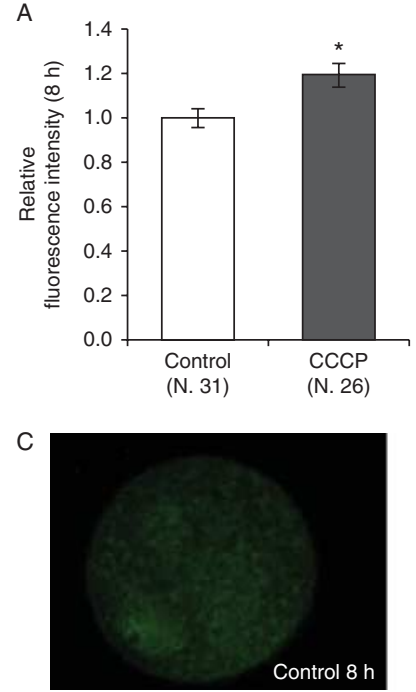
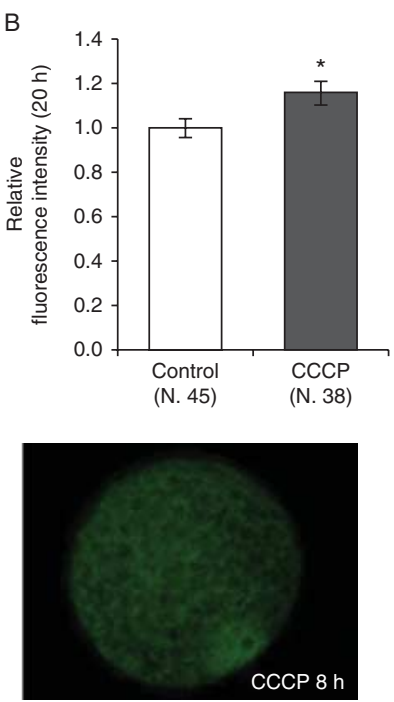

Figure 5 Effects of CCCP treatment of porcine oocytes on AMPK phosphorylation. Phospho-AMPK level were determined by immunostaining at $8 \mathrm{~h}(\mathrm{~A})$ and $20 \mathrm{~h}(\mathrm{~B})$ of IVM after CCCP treatment. Representative images of phosphorylated AMPK observed at $8 \mathrm{~h}(\mathrm{C})$. The average fluorescence intensity of the untreated oocytes was defined as 1.0. Data represent means \pm s.E.M. ${ }^{*} P<0.05$. 
with resveratrol upregulates the expression of SIRT1 and induces mitochondrial biogenesis and degradation during porcine oocyte maturation (Sato et al. 2014). In line with this, Wu et al. (2011) reported that the treatment of SH-SY-5Y cells with resveratrol activates AMPK/SIRT1 and autophagy. CCCP treatment of mouse embryonic fibroblasts has furthermore been shown to induce AMPK activation and increase the number of LC3 dots in cells (Kwon et al. 2011). In the present study, CCCP treatment of porcine oocytes was shown to upregulate phospho-AMPK by 8 and $20 \mathrm{~h}$ after treatment. In addition, we further examined the expression of SIRT1 in oocytes following CCCP treatment and found that CCCP treatment induced an increase in the expression of SIRT1 (Supplementary Figure 2, see section on supplementary data given at the end of this article). From these results, we deduce that CCCP treatment depletes ATP in oocytes, thereby increasing the AMP/ ATP ratio, which in turn activates AMPK and SIRT1, which are key factors affecting mitochondrial kinetics, including biogenesis and degradation.

In conclusion, we report that the inhibition of mitochondrial function enhances mitochondrial biogenesis and degradation in porcine oocytes. This process may play a key role in restoring and maintaining oocyte quality.

\section{Supplementary data}

This is linked to the online version of the paper at http://dx.doi. org/10.1530/REP-15-0037.

\section{Declaration of interest}

The authors declare that there is no conflict of interest that could be perceived as prejudicing the impartiality of the research reported.

\section{Funding}

This study was supported by the Promotion and Mutual Aid Corporation for Private Schools of Japan and the Ministry of Education, Culture, Sports, Science, and Technology (Grantsin-Aid for Scientific Research: S0801025) and by a Grant-in-Aid for Scientific Research C (KAKENHI, grant number: 25450400) from the Japan Society for the Promotion of Science.

\section{Acknowledgements}

The authors thank Daichi Sato for technical support.

\section{References}

Brenmoehl J \& Hoeflich A 2013 Dual control of mitochondrial biogenesis by sirtuin 1 and sirtuin 3. Mitochondrion 13 755-761. (doi:10.1016/ j.mito.2013.04.002)
Cali T, Ottolini D, Negro A \& Brini M 2013 Enhanced parkin levels favor ER-mitochondria crosstalk and guarantee $\mathrm{Ca}(2+)$ transfer to sustain cell bioenergetics. Biochimica et Biophysica Acta 1832 495-508. (doi:10.1016/j.bbadis.2013.01.004)

Chan NC, Salazar AM, Pham AH, Sweredoski MJ, Kolawa NJ, Graham RL, Hess S \& Chan DC 2011 Broad activation of the ubiquitin-proteasome system by Parkin is critical for mitophagy. Human Molecular Genetics 20 1726-1737. (doi:10.1093/hmg/ddr048)

Chen YI, Ren JQ, Kaptchuk TJ \& Kwong KK 2012 Restoring cerebral dopamine homeostasis by electrical forepaw stimulation: an FMRI study. Synapse 66 331-339. (doi:10.1002/syn.21516)

Chiaratti MR, Bressan FF, Ferreira CR, Caetano AR, Smith LC, Vercesi AE \& Meirelles FV 2010 Embryo mitochondrial DNA depletion is reversed during early embryogenesis in cattle. Biology of Reproduction 82 76-85. (doi:10.1095/biolreprod.109.077776)

Cotterill M, Harris SE, Collado Fernandez E, Lu J, Huntriss JD, Campbell BK \& Picton HM 2013 The activity and copy number of mitochondrial DNA in ovine oocytes throughout oogenesis in vivo and during oocyte maturation in vitro. Molecular Human Reproduction 19 444-450. (doi:10.1093/molehr/gat013)

Cui MS, Wang XL, Tang DW, Zhang J, Liu Y \& Zeng SM 2011 Acetylation of $\mathrm{H} 4 \mathrm{~K} 12$ in porcine oocytes during in vitro aging: potential role of ooplasmic reactive oxygen species. Theriogenology 75 638-646. (doi:10.1016/j.theriogenology.2010.09.031)

Ding WX, Ni HM, Li M, Liao Y, Chen X, Stolz DB, Dorn GW II \& Yin XM 2010 Nix is critical to two distinct phases of mitophagy, reactive oxygen species-mediated autophagy induction and Parkin-ubiquitin-p62mediated mitochondrial priming. Journal of Biological Chemistry 285 27879-27890. (doi:10.1074/jbc.M110.119537)

Ding WX, Guo F, Ni HM, Bockus A, Manley S, Stolz DB, Eskelinen EL, Jaeschke H \& Yin XM 2012 Parkin and mitofusins reciprocally regulate mitophagy and mitochondrial spheroid formation. Journal of Biological Chemistry 287 42379-42388. (doi:10.1074/jbc.M112. 413682)

Ge H, Tollner TL, Hu Z, Dai M, Li X, Guan H, Shan D, Zhang X, Lv J, Huang C et al. 2012 The importance of mitochondrial metabolic activity and mitochondrial DNA replication during oocyte maturation in vitro on oocyte quality and subsequent embryo developmental competence. Molecular Reproduction and Development 79 392-401. (doi:10.1002/ $\operatorname{mrd} .22042)$

Iwata H, Goto H, Tanaka H, Sakaguchi Y, Kimura K, Kuwayama T \& Monji Y 2011 Effect of maternal age on Mt-number, ATP content and IVF outcome of bovine oocytes. Reproduction, Fertility, and Development 23 424-432. (doi:10.1071/RD10133)

Kwon KY, Viollet B \& Yoo OJ 2011 CCCP induces autophagy in an AMPKindependent manner. Biochemical and Biophysical Research Communications 416 343-348. (doi:10.1016/j.bbrc.2011.11.038)

Lee JY, Nagano Y, Taylor JP, Lim KL \& Yao TP 2010 Disease-causing mutations in Parkin impair mitochondrial ubiquitination, aggregation, and HDAC6-dependentmitophagy. Journal of Cell Biology 189 671-679. (doi:10.1083/jcb.201001039)

Lee SK, Zhao MH, Kwon JW, Li YH, Lin ZL, Jin YX, Kim NH \& Cui XS 2014 The association of mitochondrial potential and copy number with pig oocyte maturation and developmental potential. Journal of Reproductive Development 24 128-135.

Mahrous E, Yang Q \& Clarke HJ 2012 Regulation of mitochondrial DNA accumulation during oocyte growth and meiotic maturation in the mouse. Reproduction 144 177-185. (doi:10.1530/REP-12-0113)

Mao J, Whitworth KM, Spate LD, Walters EM, Zhao J \& Prather RS 2012 Regulation of oocyte mitochondrial DNA copy number by follicular fluid, EGF, and neuregulin 1 during in vitro maturation affects embryo development in pigs. Theriogenology 78 887-897. (doi:10.1016/j. theriogenology.2012.04.002)

Pawlak P, Cieslak A, Warzych E, Zejden Z, Szumacher-Strabel M, MolinskaGlura M \& Lechniak D 2012 No single way to explain cytoplasmic maturation of oocytes from prepubertal and cyclic gilts. Theriogenology 78 2020-2030.

Pawlak P, Warzych E, Chabowska A \& Lechniak D 2014 Differences in cytoplasmic maturation between the BCB + and control porcine oocytes do not justifyapplication of the BCB test for a standard IVM protocol. Journal of Reproduction and Development 60 28-36. (doi:10.1262/jrd. 2013-092) 
Petters RM \& Wells KD 1993 Culture of pig embryos. Journal of Reproduction and Fertility. Supplement 48 61-73.

Price NL, Gomes AP, Ling AJ, Duarte FV, Martin-Montalvo A, North BJ, Agarwal B, Ye L, Ramadori G, Teodoro JS et al. 2012 SIRT1 is required for AMPK activation and the beneficial effects of resveratrol on mitochondrial function. Cell Metabolism 15 675-690. (doi:10.1016/j. cmet.2012.04.003)

Reynier P, May-Panloup P, Chrétien MF, Morgan CJ, Jean M, Savagner F, Barrière $\mathbf{P}$ \& Malthièry $\mathbf{Y} 2001$ Mitochondrial DNA content affects the fertilizability of human oocytes. Molecular Human Reproduction 7 425-429. (doi:10.1093/molehr/7.5.425)

Santos TA, El Shourbagy S \& St John JC 2006 Mitochondrial content reflects oocyte variability and fertilization outcome. Fertility and Sterility $\mathbf{8 5}$ 584-591. (doi:10.1016/j.fertnstert.2005.09.017)

Sato D, Itami N, Tasaki H, Takeo S, Kuwayama T \& Iwata H 2014 Relationship between mitochondrial DNA copy number and SIRT1 expression in porcine oocytes. PLoS ONE 9 e94488. (doi:10.1371/ journal.pone.0094488)

Shourbagy SH, Spikings EC, Freitas M \& St John JC 2006 Mitochondria directly influence fertilisation outcome in the pig. Reproduction 131 233-245. (doi:10.1530/rep.1.00551)

Smith LC, Thundathil J \& Filion F 2005 Role of the mitochondrial genome in preimplantation development and assisted reproductive technologies. Reproduction, Fertility, and Development 17 15-22. (doi:10.1071/ RD04084)

Spikings EC, Alderson J \& St John JC 2007 Regulated mitochondrial DNA replication during oocyte maturation is essential for successful porcine embryonic development. Biology of Reproduction 76 327-335. (doi:10. 1095/biolreprod.106.054536)

Takeo S, Kawahara-Miki R, Goto H, Cao F, Kimura K, Monji Y, Kuwayama T \& Iwata H 2013 Age-associated changes in gene expression and developmental competence of bovine oocytes, and a possible countermeasure against age-associated events. Molecular Reproduction and Development 80 508-521. (doi:10.1002/mrd.22187)

Tatsuta T \& Langer T 2008 Quality control of mitochondria: protection against neurodegeneration and ageing. EMBO Journal 27 306-314. (doi:10.1038/sj.emboj.7601972)

Thouas GA, Trounson AO, Wolvetang EJ \& Jones GM 2004 Mitochondrial dysfunction in mouse oocytes results in preimplantation embryo arrest in vitro. Biology of Reproduction 71 1936-1942. (doi:10.1095/ biolreprod.104.033589)

Wai T, Ao A, Zhang X, Cyr D, Dufort D \& Shoubridge EA 2010 The role of mitochondrial DNA copy number in mammalian fertility. Biology of Reproduction 83 52-62. (doi:10.1095/biolreprod.109.080887)

Wenz T 2013 Regulation of mitochondrial biogenesis and PGC-1 $\alpha$ under cellular stress. Mitochondrion 13 134-142. (doi:10.1016/j.mito.2013. 01.006)

Wu Y, Li X, Zhu JX, Xie W, Le W, Fan Z, Jankovic J \& Pan T 2011 Resveratrolactivated AMPK/SIRT1/autophagy in cellular models of Parkinson's disease. Neuro-Signals 19 163-174. (doi:10.1159/000328516)

Yoshioka K, Suzuki C, Tanaka A, Anas IM \& Iwamura S 2002 Birth of piglets derived from porcine zygotes cultured in a chemically defined medium. Biology of Reproduction 66 112-119. (doi:10.1095/biolreprod66.1.112)

Received 29 January 2015

First decision 12 March 2015

Revised manuscript received 14 May 2015

Accepted 20 May 2015 